Historic, Archive Document

Do not assume content reflects current scientific knowledge, policies, or practices. 

From the HOME of the WORLD'S FINEST

\section{Gladiolus and Dahlias}

1930

ANNUAL CATALOGUE

AND

WHOLESALE PRICE LIST

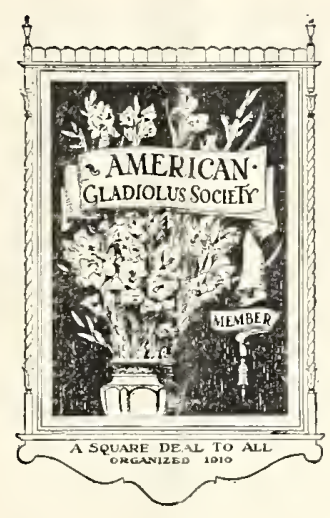

\section{A. J. ANDERSON}

White Bear Lake : : :

Minnesota 


\section{A SAVING}

For the 1930 season I have combined my wholesale and retail price lists. You will lealize this makes possible a considerable saving which I am passing along to you in reduced prices on our regular stock of high class gladiolus.

\section{DUR DOLICY OF SERVICE}

Our SERVICE POLICY in merchandising does not mean just courteous salesmanship, prompt delivery, good measure, and the like.

It consists of a great deal more than those-service in ascertaining and fulfilling the needs and desires of the public in a satisfactory and economical manner, the searching out of the best markets of the world from which may be procured the products best suited to meet our customers' requirements; merchandise which must meet and conform to the very highest standards, and which must also represent the maximum of value that is possible for us to offer at any fair price.

In fine, such service, whatever be its nature, that you would expect to come from

\section{"THE HOME OF THE WORLD'S FINEST GLADS!"}

Important-At prices quoted in this List We Deliver the Goods free to your Express or Post Office. Those who are interested in larger quantities than herein listed will please state exact amounts and I will make very low price on round lots F. O. B. here. To safeguard against disappointments later, send your orders to

White Bear Lake

\section{A. J. ANDERSON}

\section{ORIGINATORS-Including the World's Most Famous}

The names and originators of the different varieties are given using abbreviations as follows: (A) Mrs. A. H. Austin; (Bet) C. Betscher; (B) Clark W. Brown; (Boyn) Boynton; (Bill) Wm. Purple; (Br) Briggs; (Carp) Carpenter; (Col) Coleman; (Crow) J. W. Crow, Canada; (Ch) Christy; (Dames) Dames, Holland; (Doney) Doney, New Zealand; (Davis) W. B. Davis; (D) Richard Diener; (Errey) Errey Bros., Australia; (F) Eugene Fischer; (G. B. Co.) General Bulb Co.; (G) Gelser Bros.; (Gage) Lyman J. Gage; (Good) Goodrich; (Gr) Groff, Canada; (Grue) Gruellemans, Holland; (H) Hinkle; (Heemskirk) Heemskirk, Holland; ( $\mathrm{Hg})$ Dr. Hoeg; (Hop) Hopman, Holland; (Hor) F. C. Hormberger; (Houdy) Houdyshel; (J. K. R.) J. K. Rugowski; (K) A. E. Kunderd; (Kr) Keur, Holland; (Kre) Krelage, England; (Kp) Kemp; (Kel) Kelway, England; (King) Kingsley; (Kir) Kirchoff; (Kin) Mrs. Kinyon; (Lem) Lemoine, France; (Mair) Mair, Scotland; (Metz) Metzner; (Pr) Prestgard; (Pf) Pfitzer, Germany; (Par) Partain; (Pt) Pratt; (Rattray) Rattray, Australia; (S) Sanford; (Sp) Sprague; (Spr) Spencer; (St) Stevens; (U. B. Co.) United Bulb Co.; (Van M) Van Meerbeek, Holland; (Vel) Velthuys, Holland; (V. D.) Van Duersen, Holland; (Vil) Vilmorin, France; (Vos) Peter Vos; (W) Williams. 


\section{READ CAREFULLY BEFORE ORDEIRING}

The prices quoted herein are for immediate acceptance and will prevail until further notice or until my stock of 1929 bulbs are all exhausted. They include all express and postal charges for delivery to your P. O. or Express office prepaid.

My stock this year was all grown under State Inspection Permit No. 2666 and was grown on new land five miles from where any Gladioli had ever been grown-as an extra precaution, all planting stock was treated with a solution of Mercuric chloride and my stock is as clean as I have ever seen anywhere.

To foreign countries and to States that require Special Inspection Certificates I am prepared to furnish them without any additional cost.

DELIVERY - We are now prepared to fill orders at any time, weather permitting. Order's will be held for Spring delivery by depositing $25 \%$ of the amount of the order balance to be paid at shipping time or the order will be sent C. O. D. for balance if requested.

SIZES_-The sizes quoted herein are for standard sizes as recognized by the American Gladiolus Society. All orders will be filled with sizes ordered if possible. Orders for large quantities that can not be filled will be refunded. Small orders that can not be filled as ordered will be filled with the next size larger or the next size smaller, giving extra bulbs to make up the difference unless you request no substitution in your order.

5 at 10 rate, 25 at 100 rate, 250 at 1000 rate.

\section{DISCOUNTS}

For all Cash Order's received prior to March 1st, 1930, I make the following offer:

On cash orders for $\$ 5.00$ or more deduct $5 \%$ or select $5 \%$ extra bulbs.

On cash order's for $\$ 25.00$ or more deduct $10 \%$ or add $10 \%$ extra bulbs.

On cash orders for $\$ 50.00$ or more deduct $15 \%$ or add $15 \%$ extra bulbs.

On cash order for $\$ 100.00$ or more deduct $20 \%$ or add $20 \%$ extra bulbs in same or other varieties of customer's choice. It will pay you to get your friends to pool their order's with you-we will all benefit.

Minimum Order from this list is $\$ 2.00$.

Remittance may be made in Bank Drafts, Express or Postal Money Orders, or good personal checks. Please don't send postage stamps.

Guarantee-We guarantee all our stock to be true to name and free from disease. We cannot, however, assume any responsibility for crops other than to replace any bulbs not true to name or refund amount paid for same.

Reference: First National Bank, White Bear Lake, Minn.

\section{BLOOMING SIZES}

To our customers who are not familiar with the different sizes quoted herein-We do not recommend using anything smaller than No. 1, 2, or 3 for blooming the first year. Number one or Number two sizes will give best results for exhibition spikes while Number three size will give very good flowers for commercial purposes. Number 4 size may give satisfactory blooms in some localities and even the 5s and 6s may bloom under very favorable conditions, but they are usually sold only to those who wish to grow them to build up a stock for future blooms a year or two later. 


\section{WHOLESALE PRICE LIST of GLADS and DAHLIAS}

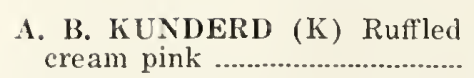

ADELINE PATTI (Kr) Dark royal purple

ADORATION (K) Lincoln reà

ADMIRATION (K) Light pink

ADA DE POY (Sal) Apricot bronze

A. E. KUNDERD (K) Rich salmon rose

AFLAIIE (Hornberger) Large prim. gr. Rose to flame red..

ALAN V. BUNCE (Bill) Orange yellow

ALIA GLUCK (Gage) Peach red with yellow throat

ALTON (K) Ruffled orange ....

ANNA EBERIUS (D) Velvety purple

ANNIE LAURIE (B) Very fine pink

ANTIOCH (Metz.) Light salmon orange $\ldots . \ldots . . . . . . . . . . . . . .$.

APPLE BLOSSOM (K) Apple blossom pink

ARABIA (H) Very dark mahogany red ..........................

ARBUTUS (Col) Arbutus pink

AUTAHI (Doney) Orange salmon purple

BATTLECREEK (K) Deep violet

BENGAL TIGER (Pt) Mottled

BER'IE SNow (Mair) Delicate lavender

BETTY NUTHALL (Sal) Clear coral pink (Gr) Bluish lavender

BLUE ORCHID (K) Violet blue
No. 1 No. 2

1
10
100
1
10
100
1000

1

10
100

100

10
100

1

10

1

10

1
1

10

100

10

100

1

10
1

10

100

1000

1
10

100

1
10

1
10

100

1
10

10

10

1

10

1

10
1

10

1
10

1
.60

…...

.30
2.50

16.00

.15

1.20

8.00

.15

1.15

8.00

.20
1.50

.60

......

1.00

.20

1.50

10.00

.10

.60

4.00

.15

1.15

.10

.60

4.00

.20

1.50

.25

2.00

.10

.80

.20

1.50

.20

1.50

1.00

.10

.75

.10

.80

1.00

8.00

5.00

.15

1.20

.15

1.20
.15
1.20

.08
.50

3.25

.25

2.00

12.00

96.00

.12
.95

6.40

.12

.95

6.40

.15

1.15

.50

4.00

No. 3

.40
2.50

.20

1.60

10.00

80.00

.10

.80
4.40

4.40

.80

4.40

.12
.90

.40

3.20

.80

9.00

.08

.50
3.50

26.00

.12

.95

.50

3.00

.15

1.25

.20

1.75

.08

.70

4.00

.15

1.25

.15

1.25

.60

.10

.80

8.00

.07

.40
3.00

22.00

.10

.80

.07

.40
2.00

.....

.90

....

1.35

.07

.60

3.00

.12

1.00

.12

1.00

.85

.08
60

.20

1.50

.08

.65

.80

6.40

.75

.50

.15
1.25

.07
.25

.50

.60

4.80

4.50

3.00

1.00

.12
1.00

.10
80
No, 4

.30

1.75

1.30

8.00

64.00

.08

.65

3.60
.08

.65

3.60

.10
.75

2.40

.50

.60

7.00

......

.70

.30

1.50

14.00

.75

3.50

1.15

.50

2.50

.75

.75

.50

6.00

......

$\cdots \cdot . .$.

.60

20

.20
1.00

9.00

.60

2.50

1.00

.40

2.00

.60

.60

.60

.40

......

.06
.35

.35
.50

4.00

.50

.......

.05

.25

40

3.60

2.50

2.00

.65

No. 6

BULBLETS

$\begin{array}{rrr}.10 & 1000 & .75\end{array}$

$\begin{array}{lll}1.00 & \text { Qt. } & 1.50\end{array}$

$\begin{array}{lll}.80 & 100 & 1.00\end{array}$

$\begin{array}{lll}5.00 & 1000 & 3.00\end{array}$

40.00 


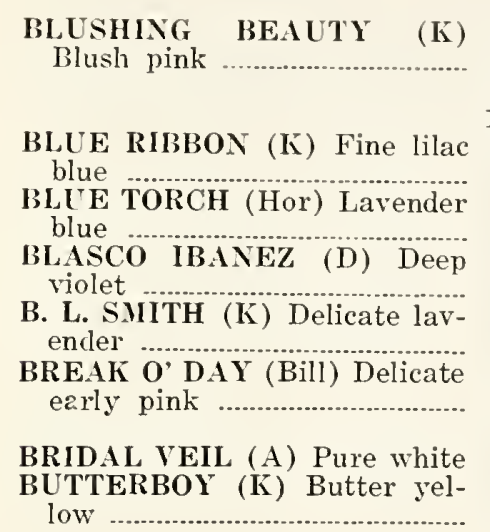

CABERNET (Metz) Wine color

CANBERRA (Errey) Pure yellow

CANANDAIGUA RED (Bill)

Bright red

CARA MIA (Miller) Lovely pink

CARDINAL PRINCE (K) Cardinal red

CARMEN SYLVA (Dec) Pure snow white

CAROLUS CLUSIUS (Vel) Fine pink

CAPT. BOY NTON (Boyn) Fine blotched lavender

CATHERINE COLEMAN (Col) Soft salmon pink

CHALLENGER (K) Large deep red

CHARLES LINDBERG (Pr) Clear hermosa pink CHARLES DICKENS (Pf) Bright radiant purple ........... CHICAGO (K) Ruffled scarlet. CHALICE F.L O W E R (K) Creamy blush pink ............... CHICADEE (Bill) Early pink. CLEOPATRA (Bill) Yellow...... Deep dark red 10 CLASSIC (K) Cream pink ........ 1 COPPER QUEEN (Metz) Bright copper CORONADO (Br) White blotched.

1
10
100
1000

.10

No. 2

$.80 \quad .65$

4.00

.15

10

1

10

1
10

10
1

10
1

1
10

100

1

1
10

100

1

10

1

1

1

10

100

1

100

1

10

1000

1
10

100

1

10
100

1
1
10
1

2.00

2.50

1.00

3.00

1.50

1
10
100

.6

.50

3.00

.15

1.25
.20

.20

.50
3.00

.40

2.50

.12
1.00

.15

.40

$\begin{array}{r}.30 \\ \hline\end{array}$

2.00

…...

3.50

3.00

2.50
No.

1.75

.10

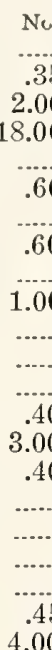

No. 4

No. 5

No.

.50

2.65

.10

.80

.75

.15

1.20

.50

.07

3.50

.60
.07

.45

.60

5.40

4.00

.......

.10
.75

. .50

4.50

$\cdots$

1.75

.45

.50

2.00

18.00

.12

.95

8.00

30

.30
1.75

.75

…....

1.80
1.00

1.00

......

.......

.75

1.75
1.00

50

.25

1.60

14.40

.50

.50

.....

…...

.30

2.25

.30

......

…-..

.35

3.00

08

.08
.50

.35

3.00

.20

1.00

.35

.40

1.50

12.00

.10

.80

6.00

.20

1.00

.20
1.50
.20

......

….

.25
2.25

......

.05
.35

.25

2.00

.15

.80

.25

.30

1.00

8.00

.60

4.00

.15

.80

$\ldots . .$.
$\ldots . .$.
$\ldots .$.
. .75
.75
$\ldots . .$.
$\cdots .$.
.60

.05
.....

10

.60

.25

.50

1.00
BULBLETS

$1000 \quad .75$

Qt. $\quad 2.00$

…. $\quad$.......

1000

$1000 \quad-75$

Qt. $\quad 2.00$

$-10 \quad .50$

Qt. $\quad 1.50$

.....

100

10

2.00

10

1.25

1000

Qt.

1000

Qt.

$$
5
$$

1000

Qt.

100

1000

Qt.

1000

Qt.

2.00

4.00

1.00

2.00

.25

1.00
2.50

1.00
4.00

10.00

1.00

5.00 
COR Y PHEE (Pf) Fine soft rose

pink

CREATORE (Bill) Salmon pink

CRIMSON GLOW (Bet) Bright red

CRINKLES (K) Extra fine ruffled cerise pink

CROWN OF GOLD (Bill) Pale straw yellow

DIABLO (Sal) Fine dark crimson

DIANA (Z) Blood red

D'S AMERICAN BEAUTYAmerican Beauty Shade

DALLAS (K) Deep salmon ....

DR. CHRIST MARTZ (K) Scarlet red

DR. ELIINS (K) Large blotched white

DR. F. E. BENNETT (D) Fine large scarlet

DR. J. H. NEELEY (K) Blushed white

DR. MOODY (Kin) Delicate lavender .................................

DR. NELSON SHOOK (K) Deep rose, extra fine .............

DON JUAN $(K)$ Delicate salmon

DR A COCEPH ALUS (Species) Greenish vellow

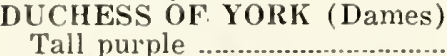
E. B. WILIIAMSON (K) Mallow purple

EARLY ONE (Bill) Begonia rose early

EARLY LASSIE (Bill) Early blushed white ........................

ED SPRINGER (K) Lavender rose pink

ELLA DAY (Carp) Fine La France pink ...........................

ELIZABETH TABOR (H) Very early blotched pink

ELIZABITH SWARTLEY (SW) Soft salmon, white throat ....

E. G. HILI (K) Fine rose pink

E.MII.E A UBRUN (Lem.) Deep smoky pink
No. 1

No. 2

No. 3

No. 4

No. 5

No. 6

BULBLETS

1
1
1
10
100
1000

2.50

2.00

1.50

1.25

1.00

.75

10

1.00

.10

.90

.80

.70

.60

.50

.60

3.50

……

1

10

100

1

10

1

1

10

100

1
10

100

1
1

1
10

10

100

1

10

100

1
10

10
100

1

10

$1 \quad .50 \quad .40$

1

1.00

5.00

.50

3.00
22.00

.40

2.50

18.00

.12

.80

.10

1.00

4.00

.70

.30

1.50

6.00

5.00

2.50

2.25

.10

4.00

.08

.65

3.25

.15

1.00

5.00

1.00

.15

1.20

......

(.....

.15

1.15

8.00

.10

.60

4.00

1.25

11.00

.10

4.00

.75

.10

.85

.........

.12

.95

6.40

.08

.50

3.50

1.00

9.00

.70

4.00

2.00

…..

2.50

.08

3.00

.50

.08

.65

.50

......

.10

80

4.40

.07

.40

3.00

.75

7.00

.30

.20

.15

1.25

1.00

.60

2.20

.60

3.00

1.50

40

2.00

.50

2.00

.......

.50

.40

2.00

.......

.70

3.60

......

2.40

.60
5

5.50

.25

.80

.50

1.40

.50

2.00

1.00

30

1.50

.40

1.50

…….

.35

.30

1.50

9.00

60

2.40

.......

2.00

.50

4.00

.20

.60

.40

.15

.20

1.00

.15

.90

.80

.12

.65

5.00

.15$$
60
$$

.10

.50

$.50 \quad .30$

.50

.40

.25

2.00

2.50

.20

1.50

2.00

.10

.80

5.00

.25

2.00

.10

....

2.00

4.00
20.00

.20

1.50

3.00

15.00

2.00

10.00

.15

.10

.10

.15

1.00

.....

1.15

1.50

80

1.00

.55

......

…..

1.00
5.00

3.50

.50

.25

....

.20

.....

1.20

.80

…...

.15

.50

1000

1000

1.50

1.00 
ENIGMA (K) Stippled lavender EUGENE LEFEBVRE (Lem) purplish Amaranth

EVELYN KIRTLAND (A)

Fine rose pink

FAIRLAND (K) Vermillion scarlet

FAY LANPHIER (W) Fine rose pink

FERN KYLE (K) Beautiful ruffled cream

FLAMING SWORD (Kel) Flaming red

FRANK M. SHICK (Sal) Clear geranium pink

FKEDERICK CHRIST (D)

Fine La France pink

FREDA (Sal) Geranium pink..

FORT WORTH (K) Shrimp pink

F.ONTAINE (Col) Large pink. FRANK J. McCOY (Br.) ........
GAY HUSSAR (Sal.) Salmon orange red REALDINE FARRAR (D) Lavender blue GERTRUDE PFITZER (Pf)

Fine new violet ..................

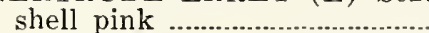

GIANT MYRTLE (K) Fine large pink ...............................

GIANT NYMPH (Col) Fine large pink

GLADDIE BOY (Bill) Salmon orange

GLENDALE (Davis) Dark violet

GLORIANA (Bet) Golden salmon
OLD (Hg) Pure golden yellow
.20

1.50

6.00

$\cdots . .$.

.80

4.00

.10

.70

.10

.80

.10

.70

4.00

35.00

.15

1.00

5.00

40.00

1.00

3.00

.25

.75

.10

-.....

2.50

1.00

1.20

5.00

.09

.70

3.00

.08

.60

4.00

.08

.70

.08

.60

3.50

30.00

.12

.80

4.00

32.00

.90

2.75

.20

.60

.08

.60

2.25

.80

.20

.25

.30

1
1
1
10
100

1000

1

10

100

1000

1
10

100

1

10

100
1000

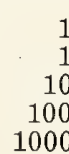

2.00

.15

.10

......

.....

.10

.60

4.00

.25

2.00

9.00

.10

.50

4.00

......

.30
.10
.80
4.00

4.00

.12

.70

3.00

27.00

.08

.50

3.50

27.00

.20

1.75

7.00

.08

.40

3.00

27.00

.25

.60

3.50

32.00
…... .15

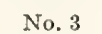

.10

.80

4.00

.08

.50

2.40

$-50$

3.20

.60

.07

.50

3.00
25.00

25.00

.10

.70

3.20

26.00

.75

2.00

.15

.50

.07

.50

2.00

.70

.15

.20

.10

.60

2.40

22.00

.07

.40

3.00

22.00

.15

1.25

4.00

.07

.30

2.40

22.00

.20
.07
.50

24.00
No. 4

.08

.60

3.20

-.....

.40

1.80

40

2.60

.50

......

.40

2.00

18.00

.55

2.60

20.00

.60

1.50

...

.40

1.75

.30

1.50

1.50

.50

.35

1.00

1.00

.20

.05

15

No.

No. 6

.05

.25
$50 \quad 1.50$

20.00

12.00

.20

.80

.20

1.50

.30

....

.20

1.00

8.00

.30

1.50

10.00

.40

1.00

......

1000

1.00

10

1.00

10

.75

.05

.40

1.80

16.00

.30

2.25

16.00

.10

1.00

3.00

.20

1.80

16.00

.45
. .30
1.20
11.00
$\ldots$.
.20
1.25
11.00
.7
.75
. .00
.15
1.20

.25

.20

7.00

.10

.10

.75
7.20

.50

1.00

.

.10

.80

11.00

7.00

.15
$\ldots$.
.30
1.80

.10
$\cdots .$.
1.20
1.20

18.00

.10

8.00 
GOLD EAGLE (A) Rich yellow

GOLDEN BUTTERFLY (K)

Fine yellow

GOLDEN DREAM (Gr) The very best deep yellow ............

GOLDEN MEASURE (Kel) Large tall yellow

GOLDEN SALMON (K) Salmon yellow …………........

GOLDEN SWALLOW (A) Very fine yellow

GOLIATH (Vel) Deep purple....

GOSHEN (K) Rose pink blotched red yellow prim ..............................

GOLDEN GLOW (Hg) Yellow,

HALCYON (Bill) Blotched pink

HARBINGER (S) Fine burnt Orange lavender blue

HELGA (K) Good salmon rose

HENRY FORD (D) Dark velvety purple

HELEN HOWARD (A) Golden buff shade and earlier than W. H. Phipps

HELEN WILLS (Sal) White,

$\begin{array}{ll}\text { cream throat . } \\ \text { H E A D A } & \text { (A) Glistening }\end{array}$ mauve

HINEMOA (Doney) Pink flaked chocolate

HONEY DEW (Pr) Laciniated orange

ILLUNIINATOR (G. B. Co.) Tall fine red .........................

IMMENSITY (K) Large salmon pink

INDIAN SUMMER (K) Large lavender pink late

\begin{tabular}{|c|c|c|c|c|c|c|c|}
\hline No. 1 & No. 2 & No. 3 & No. 4 & No. 5 & No. 6 & \multicolumn{2}{|c|}{ BULBLETS } \\
\hline .20 & .18 & .15 & & & & $\ldots \ldots$ & \\
\hline 1.60 & 1.20 & & 60 & .50 & .40 & & \\
\hline & & & 5.00 & 4.00 & 3.00 & 1000 & 2.50 \\
\hline .10 & .08 & .07 & & & & & \\
\hline .80 & .65 & .45 & .30 & .20 & .15 & 1000 & 1.00 \\
\hline & & & 2.50 & 2.00 & 1.20 & Qt. & 2.00 \\
\hline .40 & .35 & .30 & .25 & .20 & .15 & 100 & 1.00 \\
\hline 3.20 & 2.75 & 2.25 & 1.75 & 1.25 & .75 & 1000 & 8.00 \\
\hline 20.00 & 15.00 & 12.00 & 00 & 8.00 & 5.00 & Qt. & 25.00 \\
\hline 0.00 & 120.00 & 0.00 & 75.00 & 60.00 & 40.00 & Pk. & 150.00 \\
\hline .10 & .08 & .07 & ..... & ....... & ...... & & \\
\hline .65 & .55 & .45 & ....... & ....... & ....... & 1000 & 1.00 \\
\hline 4.00 & 3.00 & 2.50 & ....... & ....... & ....... & ....... & .... \\
\hline .25 & .20 & .15 & .10 & .08 & .05 & 1000 & 2.50 \\
\hline .20 & 15 & & ...... & ….. & $\ldots . .$. & ...... & $\cdots$ \\
\hline$\cdots$ & 1.25 & 1.00 & 300 & ת & & & \\
\hline 10 & 0.00 & 4.00 & 3.00 & 2.00 & 1.00 & 1000 & 1.50 \\
\hline .90 & .75 & .60 & .50 & ....... & $\cdots \cdot$ & …... & $\cdots \cdot$ \\
\hline 4.00 & 3.00 & 2.50 & 2.00 & ....... & ....... & ....... & ... \\
\hline .1 & .12 & .10 & & ........ & . & & \\
\hline 1.00 & .75 & .60 & .50 & .30 & .20 & 100 & 1.00 \\
\hline .25 & . & .15 & .10 & & & & \\
\hline 2.00 & 1.20 & 1.00 & .80 & .50 & .25 & 100 & .50 \\
\hline
\end{tabular}

$1 \quad .25$

$.20 \quad .15$

.15

6.00

.20
1.20

.80

5.00

.15

1.00

1.00

.80

.10

10
100

1

$10 \quad .80$

3.30

.08
.60

4.00
32.00

1000

3.00

24.00

.45

1.50

10.00

100

.75

.10

.50

3.40

1.25

9.00

65.00

.60

.10
.70

4.00

.10

.50

.40

3.00

2.00

.20

1.00

1000

1.00

.45

.30

1000

2.00

.60

.07
.40

3.00

.07

2.75

.30

2.40

1.00

8.00

55.00

.50

.50

$\ldots . .$.
$\ldots . .$.
$\ldots .$.
1.00
$\ldots . .$.
$\cdots \cdots$.
$\cdots \cdots$
...
.10
.80

1.60

.75

6.00

45.00

.40

4.50

35.00

.30
1.50

.40

....

2.50

1.50

......

.20
1.20

1.50

2.50

2.25

2.00

1.75

.80

100

4.00

1000

75

1.00

$.30 \quad 25$
.00

.10

.80

.10

.75

.08

.70

.08

.60

$\cdots . .$.
$\cdots \cdots .$.
$\cdots . .$.
.60
$\cdots \ldots$
.50
3.00

1.50

1.25

1000

......

.75

.50

100

.75

3.00

25.00

.25

1.00

…....

.50

1000

10

2.00

$\ldots . .$.
$\ldots . .$.
.40
$\ldots . .3$
.35
2.00
15.00

$\ldots . .$.
$\ldots . .$.
...
.30
$\ldots \ldots .$.
.25
1.00
8.00

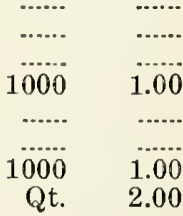

.12

.10 
IW A (Bet) Light salmon pink, very large

J. A. CARBONE (D) Large orange salmon ....

JACK LONDON

(D) Salmon striped red

JACOB A VON BEYEREN (V D.) Dark violet

JANE ADDAMS (Pr) Soft phlox pink

JAP LADY ( $\mathrm{Pr}$ ) Bishop purple

JENNY LIND (H) Lovely coral pink

JEWELL (Z) Pink, yellow throat

JOERG'S WHITE (J) Extra large white

JOERG'S YELLOW PRIII, Fine large yellow

JOHN TOLAND (Sal) Blotched peach red

JOHN T. PIRIE (K) Mahogany brown blotched

JOY

(K) Delicate creamy pink

JOHN F. PEABODY (Metz) Deep rose pink

JOSEPH FIELD (D) Light pink cream throat

J. H. McFARLAND (K) Ophelia rose color

KATHERINE HESS (D) Geranium pink

IING GEORGE (Mair) Dark

red with cream blotch .-......
KING OF. BLUES (Gr) Deep purple

KIRCHOFF'S VIOLET (Kir) Pure violet - -... VW VIOT (Kir) Deeper violet ................

LACINATUS (K) Laciniated pink

LA COURONNE (Lem) Yellow with a fine large red blotch....

LAVENDER PRINCESS (W) Lavender cerise .............................

LE CYGNE (Houdy) Large white prim light lavender
No. 1

1

10

100

1
10

100

1

10

10

100

1

1

10

100

10

100

1000

10

1

10

10

1

10

1000

1

10

1

1

1

1
10

100

1

10

1

10

1

1

10
100

100
1

10

1
10

1

1
10

100

.15

1.20

.10

.60

.10

.50

.10

.75

.50

.10

.70

5.00

.10

.80

5.00

40.00

.50
4.00

4.00
.50

4.00

.35

2.50

.10

.80

6.00

.15

2.00

.10

.50

1.25

$\begin{array}{r}.25 \\ \hline\end{array}$

.25

.40

.10

…..

1.50

.20

2.50

.15

1.00

8.00 $\begin{array}{ll}.15 & .12 \\ \ldots . . . & .80\end{array}$

.25

.30

.25

1.50

.08

.40

.07

.30

2.40

1.00

.20

.75

1.60

.20

1.75
.35

.08

.60

$20 \quad \cdots$

1.25

1.50

2.00

.10

.15

1.20

.15

1.40

.30

.07

.50

.15

1.00

1.00

.80

1.50
-.60
6.50

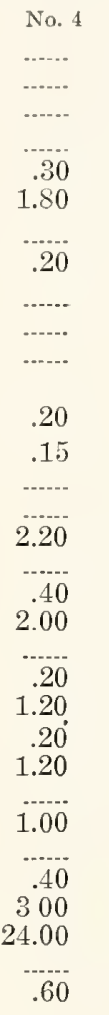

No.

No. 6

BULBLETS

200

1.00$$
.20
$$

1.00

1000

1.50

1.20

.10

.10

-..--...

1000

1.00

1000

.50

...-...

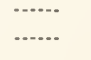

.15

.10

.10

1.40

1.0

.25

1.00

$-.-15$

.15
1.15

.15

1.15

.75

... .8
.30

200

16.00

.10

.1

.10

.10
.80

.10

.80

.50

.20

1.00

.40

-----

.05

100

2.00

.75

.50

1.80

.40

1000

.50

.40

.75

.10

.90

.20

.05

.40

.10

.60

.40

1.25

.80

.60

.25

.50

.25

.05

.30

100

---.

80

.80

1000

...-.

-----

.80

.50
.35
.35
3.00

...

.50
4.00

3.00
10

Qt.

10

100

1.00

100

1.00

1.00

1.00

1.00

-.-...

2.00

-..--

1.00

-.--

\begin{tabular}{rr}
10 & 2.00 \\
$\cdots \cdots .$. & $\cdots . .-$ \\
\hdashline 100 & $1 .$. \\
\hline
\end{tabular} 
LILLIAN HARRIS COFFIN

(D) Shrimp pink ................. 10

LILYWHITE (K) Early white

1000

LOA LUVERNE (K) Salmon 1 and lavender pink .................. 10

LONGFELLOW (Pr) Extra fine 1 pink ................................... 10

LOS ANGELES (Houdy) Gren- 1 adine pink

LOYALTY (A) A very fine yellow . SULLY (Lem) Ivory with blood red blotch

MADAM NORENA (Pr) Light flesh pink ..............................

MAINE (Vos) Tall white ........

MAJESTIC (Hop.) Orange scarlet

$$
\begin{array}{rr}
10 \\
100 \\
1000
\end{array}
$$

MAMMOTH WHITE (U. B. Co.)

MANDARIN (P) Salmon orange, very fine .................. Each

MARY LOCKWOOD (Kin) Fine early pink

MARIE K U NDER D (K) Very fine, white

MARIETTA (Metz) Geranium pink

IIARION KOCH (A) Pure rose pink

MARMORA (Errey) Rich smoky lavender

MARNIA ( $\mathrm{Kp}$ ) Orange

MARSHAL FOCH (K) Salmon pink

MARY FREY (G) Fine light lavender

MARY JANE (K) Lavender

Mink ${ }_{\text {M }}$ S. BURKE (D) Primrose yellow

MASTLRPIECE (K) American beauty color.

1
1
10
100
1000

1000

10

100

10

1

10

100

1

100

1

10
100

1000

ro. 1

.20
1.50

.50

2.50

20.00

.75

.20
1.50

.20

.10

.60

4.00

32.00

1.50

12.00

.30

2.40

.75

.10
.90

4.00

.10

.80

5.00

6.00

50.00

.30

2.00

.10

.75

5.00

40.00

.10

.80

5.00

1.00

8.00

1.00

8.00

.15

1.00

6.00

.10

.65
4.00

1.75

.08

.60

4.00

32.00

.08
.70

4.00

.85

7.00

.80
6.00

.......

.12

.80
5.00

.08

.55
3.00

1.25

1.00

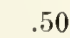

.15

100

100

10
100
.15

1.20
.40

.12
.80

4.00

.12

1.00
No. 3

.....
$\cdots . .$.
.30
.50

1.50

.......

......

1.00

1.00

.07

.40

2.40

19.20

1.00

8.00

.20
1.00

1.60

.50

.50

2.40

.50

3.00

4.00

30.00

1.25

.50

3.00

24.00

.07

.60

3.00

.75

6.00

.60

4.50

.10

.60
4.00

.07

.45

2.40

1.00

.30

2.20

17.50

.50

2.20

.65

5.00

.40
3.00

25.00

$\cdots . .$.

.45

....

1.80

.80

.60

.30

.10
.75

3.00

.10

.80

.25

2.50

.60
No. 5 No. 6

…....

........

.......

…...

.......

.45

.45

.20

1.20

9.60

.50

4.00

.......

.20

......

…...

.20

1.00

7.50

2.00

15.00

.10
$\ldots . .$.
$\ldots . .$.
$\ldots . .$.
...
.10
.75

5.00

1.00

8.00

1000

10

100

30.00

.75

.50

.20

1.40

12.00

.40

1.40

.60

4.50

.30

2.50

20.00

30

2.30

.20

.20
1.20

7.00

.10

1.00

8.00

.30

1.00

.50

3.50

.20

2.00

15.00

.....

1.00

.....

.80

6.00

.50

.40

.20

.15

.......

200

1.50

1000

10

1000

1.50

…........

4.00

…....

2.50

Qt.

1.00

2.00

1000

1.00

5.00

$\begin{array}{rr}100 & 1.50 \\ 1000 & 10.00\end{array}$ 
MAURICE FULD (Gage)
Large rose pink .....................
MAROLEE (Carp) Jasper pink
with yellow blotch .............. with yellow blotch
IILADY IMOGENE (A) Snow white prim Gr.

MING TOY (K) Buff yellow ...

MINUET (Col) Delicate lavender pink

MISS DALLAS (K) Sea shell pink ruf.

MISS DES MOINES ( $\mathrm{Pr}$ ) Clear pale lilac

MISS MADISON (Boyn) Clear light pink

MOTHER MACHREE (St) Smoky prize winner ............. MR. H. A. HYDE (D) Snow white blushed pink

(D) Snow

IR. W. H. PHIPPS (D) Fine exhibition var., soft pink .....

IRS. ARTHUR MEEKER (K)

Am. Beauty Rose

$1 \quad$ No. 1 100

1000

1000

MRS. CATHERINE EDWARDS (Horn) Early creamy white...

MRS, C. F. HORNBERGER (Hor) Pure white

MRS. F. C. PETERS (F) Tall fine lavender

MRS. J. K. ARMSBY (D) Coral pink, very large

(D) Cor-

IIRS. J. R. WALSH (D) Ruffled, flesh pink, blotched ....... 10

MRS. JOHN S. WOOD (Metz) Similar to Mrs. L. Douglas...

MRS. KRISTIAN PRESTGARD (Pr) Pure snow white

MRS. LEON DOUGLAS (D) Begonia rose sriped scarlet...

MRS. N. VANDERBILT (D) Salmon rose pink ruf. ....... MRS. P. W. SISSON (Col) Cameo pink .... Orange salmon
10

2.75

No. 2

No. 3

.25

.20

.....

8.00

4.00

5.00

.50

4.00

.40

3.20

30
.30

.30
2.40

.08

.80

4.00

.65

3.00

.50

2.40

.75

.60

.50

5.00

4.00

1.00

8.00

.15

1.00

5.00

......

25.00

.15

1.00

6.00

.15

1.15

12.00

.10

.80

$\ldots$

7.00

4.00

.10
.90

4.00

32.00

.08

.70
3.00

24.00

3.00

.50
2.00

16.00

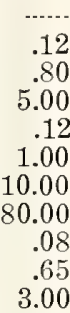

.10

.70

4.00

.10

.80

8.00

64.00

.50
.50

2.40

No. 4
.10
.90
6.00

2.00
.20
1.60
$\ldots .$.
.30
$\cdots-.$.
.40
3.00

No. 5

No. 6

BULBLETS

.08
.70

4.00

.50

1000

3.00

1.00

.60

.15

.10

10

100

.20

.15

.30

.20

1000

100

2.00

25.00

.30

2.00

15.00

.10

.80

4.00

.12
1.00

1.00
5.00

5.00

.10

$\begin{array}{lr}\ldots . & .65 \\ \ldots . . & 4.00\end{array}$

.15

1.00

20.00

.25

1.70

12.00

.08

3.20

.10

.90
4.00

15.00

.20

1.50

10.00

.07

2.40

.08

.75
3.00

.50
2.20

.......

16.00

.30

1.40

11.00

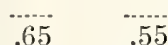

3.00

2.00

.80

.10
.70

$-. .$.

1.00

.40

.12

.20

.15

.10

.08

.07

.60

.40

$.12 \quad .10$

.15

$10 \quad 1.15$

1.00

.30

.90

.20

3.20

2.40

1.60

........

…....

1000

1.00

1

1.00

.50

1.00

1000

3.00

.50

.40

1000

2.50

32.00

2.00

.2

.30
1.20

9.60

6.40

1000

1.00

$7.50 \quad 5.00$

.12

5.00
.10

.75
3.00

Qt.

1

100

1000

1000

Qt.

1000

1.00

1000

8.00

.......

1000

1.00

.30

1000

1.00

.10

$\ldots$

100

\begin{tabular}{|c|c|c|c|c|}
\hline .60 & .40 & .30 & .20 & \\
\hline & …... & -..-. & $\cdots$ & 1000 \\
\hline .90 & 75 & 60 & 40 & 1000 \\
\hline .20 & .15 & .10 & & \\
\hline 1.60 & 1.20 & .80 & .50 & 1000 \\
\hline
\end{tabular}

10 
A. J. ANDERSON, White Bear Lake, Minnesota

MRS. T. RATTRAY (Rattray)

Rose pink, many open ...........

IIRS. RICHARD LOHRIIAN

(D) Shell pink

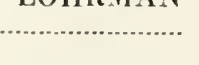

MRS. ROBERT CUNNING-

HAMI (D) Cameo pink ..........

IIRS. YON KONYNENBURG

(Pf) Fine tall lavender ........

IIURIEL (Pf) Fine tall lavender

NANCY HANKS (Sal) Orange pink

NATALIE (K) Deep orange salmon ...................................................

OH BOY (Bill) Large brilliant red

ONEIDA JANE (Carp) Geranium pink early ..................

OPALESCENT (Bill) Lavender pink

OLIVAR (Errey) Violet blue...

ORANGE BU'TERFLY (Sal) Olange bronze ...................... million orange

ORANGE QUEEN (Pf) Fine orange prim.

ORCHID LADY (Spr) Early Orchid lavender.

PAPILIO (D) Shrimp pink .....

PARAMOUNT (K) Most beautiful pink

PATRICIA CARTER (K) Very delicate pink

PAULINE KUNDERD (K)

Fine pure rose pink
PAUL PIITZER (Pf) Velvety purple $\ldots$ ROSE (K) Fine rose pink

PEARL OF CALIFORNIA (King) La France pink ....... 10

PERSIA (N. B. Co.) Very dark red

PFITZERS TRIUMPH (Pf)

Bright salmon orange
PHINI (D) Cameo pink
No. 3

No. 4

No. 5

No. 6

BULBLETS

1
1
10
100
1000

2.00

.20

1.25

5.00

.35

2.50

.60

4.80

.10

.90

4.00

32.00

.20

1.00

6.00

.15

1.00

3.00

30

2.00

.50

4.00

.80

3.00
24.00

.15
.80

5.00

.12
.80

2.40

19.00

.25

1.50

.40

3.20

.50

2.40

19.20

.10

.60

3.00

.15

.10

.12

1.50

1.25

1.00

1

10

100

1000

.10

.65

4.00

30.00

1.00

.90

.08

.55
3.50

25.00

$\begin{array}{rr}1 & 1.00 \\ 1 & .15\end{array}$

$10 \quad 1.15$

100

9.00

.10

.60

4.00

32.00

.50

2.50

.40

3.50

.20

1.60

.12

.90

6.00

.08

3.00

$24.00 \quad 20.00$

.40

2.00

3.00

.15
1.20

6.00

.40

.50

3.50

.10

.60

4.00

.50

3.00

$24.00 \quad 19.20$

.70

5.50

.10

.70

10
100

.08

.60

.65

5.25

.55

4.50
1.00

.80

.07

.45

3.00

.85

20.00

.70
4.00

.07

.40
2.40

20.00
.30

1.75

.30

2.75

.90

4.00

.50
3.00

.35

.25
1.00

1000

Qt.

1.50

.30

1.80

.20

14.40

1.20

.10

.80

1000

6.40

1.50

9.60

1.00

.75

2.00

1.50

1.00

.60

.50

3.00

2.00

.40

1.00

.50

.40

.30

.20

10

Qt.

100

1000

100

1000

3.00

2.50

2.00

.30
.50

.25

.20
1.50

1.50

10

. .50
.07

.40

2.40

2.00

.30

1.80

.20

14.40

1.20

9.60

.50

.40

.30

2.50

2.00

16.00

.07

.50

4.00

.45
3.75

.40

3.00

.30

2.00

.20
1.60

$2.20 \quad 1.60$

$.30 \quad .20$

.15

1.00

100

.10

.80

6.40

.20

1.50

12.00

.20

1.00

.10

1.10

.15

1.00

1.00

1.00

1.50

1.25

$.60 \quad .50$

100

Qt.

100

1000

2.40

20.00

10.

1000

100

1.00

$15 \quad 1.50$ 
PINK CLOUD (K) Fine ruffled

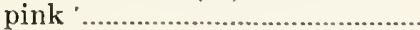

PINK WONDER (Kp) Large pink

PRES. HARDING (Par) Deep tyrian rose ..............................

PRIDE OF LANCASTER (K)

Rosy salmon ruf. ...................

PRIMI BEAUTY (Ch) Rich salmon buff

PRIDE OF HILLEGOM (Vel) Bright red

POLYANNA $(\mathrm{Hg})$ Good ruffled yellow

PRIMROSE PRINCESS (Sal) Primrose yellow .....................

PUREST OF ALL (Pf) Fine pure white

PURPLE GLORY (K) Garnet red ruf., extra fine ...............

PURPLE QUEEN (K) Violet purple MARY (Sal) Live strawberry pink

QUEEN MARY (Mair) Creamy white flaked with rose pink

RADIANT ORANGE (A) Bright orange

RED EMIPEROR ( $\mathrm{Gr}$ ) Fine red

RED FIRE (K) Fiery scarlet..

REAH (Sal) Rich mallow purple

REV. EUBANK (Vel) Light lavender

RICHARD DIENER (D) Salmon pink

RITA BECK (F) Pure shell pink OBERT J. KUNDERD (K) Fine large red

ROALD AMUNDSEN (Pr) $\begin{array}{lc}\text { Spectrum red .................. } & \text { (K) Salmon rose }\end{array}$ mottled
No. 1

No. 2

No. 3

No.

1

1

10

100

100

1

10

1

10

100

1

100

1

10

100

1

1

10
100

1
10
100

100
1

1
10

1

10

1

1
10

10

10

100

1000

1
10

1
10

10
100

1

10

1000

1

10
1

10

100

1000

$\begin{array}{ll}1 & .75 \\ 1 & .10\end{array}$

10

100
.10

.80

.15

1.20

6.00

.10

.75

.10

.75
6.00

6.00

.10

4.50

.10

.75

.75

2.50

.25

2.00

.10

.70

6.00

.25

2.00

.30

......

2.00

.75

2.25

2.00

.10
.70

4.00

35

2.50

.10

.65

4.00

.10

.65
4.00

.75

6.00

.10

.80

5.00

.75
.10
.60
4.00

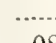

.08

.60

4.00

.12
.90

5.00

.08

.08

.08

.60

5.00

.08

.60
4.00

.08

.08
.60

.60

2.00

.20

1.50

8.00

.08

.60
5.00

.20

1.60

.25

2.00 .

1.50

.65

.20
1.50

.60

3.00

24.00

.30

2.00

.55

3.00

.08

.55

3.50

26.00

.60

5.00

.70

4.00

32.00

.60
. .50
.500 $\ldots . .$.
.50
.50
.10
.60
4.00

.07

.50

.07

.50
4.00

4.00
.07

.50
2.50

.07

.50

.50

1.75

.15

1.20

6.00

.07

.50

4.00

.15

1.20

.20

1.50

1.25

.55

.15

1.00

.50

2.40

20.00

.25

1.50

.45

2.40

.07

2.75

22.00

.40

3.60

.....

3.00

24.00

.50

40

2.40
.07

.30

......

.40

3.00

.40

.40

3.00

1.75

.40

1.80

.35

1.50

.10

.80
4.00

.00

40

3.00

.10

.80

1.00

1.00

.45

.10

.80

.40

1.80

14.40

.20

1.00

......

$\ldots . .$.
$\ldots . .$.
.35
2.00

16.00

.30

2.40

.40

2.20

18.00

30

1.20

.25

……

2.75

30

2.00

.60

.80

.35

.60

.30

1.20

11.00

.15

.....

......

1.5

12.00

.25 
ROSE (K) Ruffled rose pink....

ROSE GLORY (K) Rose pink..

ROSE MIST (F) Watermelon red prim.

ROSE PRECOSE (U. B. Co.)

Rose salmon pink

ROZAN (A) Delicate rose pink

RUBINI (Heemskirk) Bright salmon scarlet with pure white throat

R UFFLED GOLD (Goodr) Lovely golden yellow

RUFFOLACE ( $\mathrm{Kp}$ ) Laciniated white .............................

ROYAL, CROWN (Metz) Clear lilac purple

RUTH HUNTINGTON (K) Lilac pink

RUTH TAYLOR (D) Extra

large white

SALBACH'S P I N K (Sal)
Large glowing pink

SA MATEO (Sal) Clear rose pink

SANS PAREIL (Vil) Apricot pink ….................................

SCARLANO (K) Tall scarlet..

SCARLET BEDDER (Sal) Glowing scarlet prim. ............

SCARLET P'RINCEPS .... (K) Scarlet self

SCARLET WONDER (Gr) Known as Cowie's scarlet wonder. Fine scarlet..............

SEBASTA (K) Blushed white.

SENATOR KNOX (K) White with blotch

SENORITA (Sal) Orange salmon

SERRATA (Bill) Creamy yel-

SHIELA (Col.) Early light salmon

SHELL, PINK (K) Light rose pink

SHEBA (Sal) Tall early pink. SHOW FLOWEIR (K) Red with white throat
No. 1

1

10
100

1

100

1
10

100

1
10

100

1

1
10

100

1
10

10

1
10

1

10

Each

1

1

1

1

100

10

1

10

100

1

10

100
1000

1

100

1

10

1
1

10

10

100

10

100

1000

1

1
10

\section{.10}

1.00

7.00

.10

.60

4.00

.20

1.20

9.00

.15

1.15

3.00

.15

1.00

7.50

2.00

16.00

.50

4.00

2.00

.10

.60

1.00

5.00

1.00

.10

.60

4.00

.25

1.50

.10

.70

4.00

.10

.60

......

.10

.60

5.00

.15

1.00

.25

2.00

.10

.60

4.00

.10

.60

4.00

32.00

.50

.25

1.50
No. 2

.90

6.00

.50

3.00

.15

1.15

8.00

.12

1.00

8.00

2.50

.12
.80

6.50

1.50

12.00

.40

3.00

1.50

.50

.90

4.50

1.00

.85

.20

.08
.50

3.00

.20

1.25

.08
.60

3.00

.50

3.00

24.00

.08
.50

4.00

.12

.90

2.40

19.20

3.00

.90

.20
1.75

1.00

.50

3.00

.50

3.00

.40

2.40

24.00

.40

.20
1.20
3.50

2.00

1.50

.15

.10

.07
.40

2.40

.15

1.00

.07

.50

2.40

.40

.40

.10

.80

.40

2.40

No. 4

.50

3.00

.30

1.80

.70

4.00

.65

6.00

1.50

.50

4.00

.75

6.00

2.00

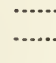

.30

1.80

.90

.40

1.80

.30

1.50

14.40

.30

2.00

.60

$\ldots \ldots$.
$\ldots \ldots$
.20
1.20
$\ldots \ldots$
.75
$\ldots \ldots$.
.30
1.20
$\cdots \ldots$.
.20
$\ldots \ldots .$.
$\cdots \cdots$
.20
1.00
$\cdots \ldots$
.50

.50

.60

.50

80

.60

.30

1.80

.20

1.20

.30

1.80

.20

$19.00-15.00$

.25

.15

1.00
No. 6

30

1.00

.10

.80

.35

35

4.00

.30

2.00

BULBLETS

1000

1.00

1000

2.00

1000

2.00

10

2.00 
SIBYL (Bill) Creamy pink........ 1

SIDNEY PLUMIIER (Sal) Soft 1 yellow with pink tips

SNOW BOY (K) White with 1 throat marking ....................... 10

SNOW GLORY (K) Ruffled

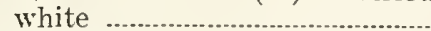

SOUVENIR (Gr) Deep yellow prim

SULPHUR FRILLS (K) Light sulphur yellow ruffled

SUPERBA (Col.) Carmine scarlet

SWEET LAVENDER (Col) Early lavender

SWEET ROSE (K) Rose pink

SYLVIA WES'T (J. R. R.) Purest white

TARO (K) American beauty shade -...............................

$\underset{\text { pink }}{\text { THEDA (Bill) Early shrimp }}$

THAIS WALDEMAR (D) Vermillion rose ..................... THOMAS A. EDISON (K) Dark wine red

TIGER (K) Smoky mottled....

THE ARISTOCRAT (Bill) Saffron pink flaked orange ........

THE ORCHID (Sp) Orchid color prim.

THOMAS T. KENT (D) Rose pink blotched

TITANIC (Hg) Large dark red

TOBERSUN (A) A new pure yellow

TREASURE (K) Salmon rose pink 10
100 10 100 1000 100

1

1

100

1000

10

1

10

100

1

10

100
1
10

100 1000

No. 1

.10

.65
5.00

.15

.75

5.00

.15

1.00

5.00

.25

.10

.60

.....

.15

.15

80

4.00

.15

1.25

.10

.60

4.00

.25

2.00

100

1

1
10

10
1

10

100

1000

1

10

1

1
10

1

10
100

1

10

100

1
10

100

1000

1
10

100

1

1
10

.20

1.00

.08

.80

5.00

.15

1.25

5.00

.30

2.50

.25

2.00

10.00

.60

......

.10

.75

4.00

32.00

.10

.80

5.00

2.00

2.00

1.00
No.

.50

4.00

.10

.60

4.00

.10

.80

4.00

.20

.50

3.00

......

.10

.10

60

3.00

.12

1.00

.08

.50

3.00

24.00

.20
1.60

8.00

.40

3.00

.50

3.00

……

.60

3.00

.15

.40

2.40

20.00

.08

.08

50

2.40

.10

.80

.07

.40

2.40

19.00

.15

1.20

6.50

.15

.12

.90

.07

.60

4.00

.12

1.00

4.00

.25

2.00

.20
1.75

1.75

9.00

.50

4.00

.......

3.00

24.00

.08
.70

.70
4.00

1.75

.12

.40

3.00

24.00

.10

.80

3.00

$\begin{array}{r}.20 \\ \hline\end{array}$

1.60

.15

1.40

8.00

.40

3.00

......

.50
2.40

20.00

20.00
.07

.60

3.50

1.50
No. 4

No. 5

18.00

.50

2.00

.10

.30

1.80

14.40

.06

.06

40

1.80

.60

5.00

30

1.80

14.40

1.00

5.00

40.00

60

.60

.30
2.00

16.00

.60

2.50

1.25

1.00

1.00
5.00

.30
2.00

.40

1.80

15.00

....

2.50

1.25

1.00

.60

.30

1.40
12.00

.40

1.50

.08

.20

1.20

10.00

.05

.05

.30
1.20

.50

4.00

.20

1.20

11.00

80

4.00
.30
...-
.40
2.20

No. 6

BULBLETS 
TRIUMPHATOR (Kre) Extra

large red

TUTANEKAI (Doney) Darker sport of Hinemoa ....................

TWILIGHT (K) Large creamy pink

TYCO ZANG (A) Large salmon pink

TYRIAN BEAUTY (K) Tyrian rose pink

VANITY (Bill) La France pink

VEILCHENBLAU (Pf) Dark violet blue

VICTOR (Errey) Bright scarlet with white blotch ..................

VIOLET GLORY (K) Ruffled violet

$$
\begin{array}{r}
1 \\
1 \\
10 \\
100
\end{array}
$$$$
1000
$$

VIRGINIA HALE (K) Rich creamy pink

WATERMELON (K) Vermil-

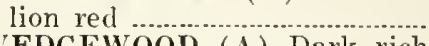

WEDGEWOOD (A) Dark rich violet ........................... Large white

WHITE ORCHID (Bill) Fine white ing white

WHITE WONDER (Kp) Large white

WILLIA M BADGER (Metz) Fine tall pink

WILLIA.M KEN'T (D) Fine creamy pink

YELLOW GLORY (K) Deep yellow with pink blotch .......

YELLOW PERFECTION (Pf) Pure soft yellow

100

1
1
10
100

1000

1
10

1000

1
10

100

10

100

1

10

$\begin{array}{cc}\text { No. } 1 & \text { No. } 2 \\ .35 & .30 \\ 2.50 & 2.00 \\ 20.00 & 16.00\end{array}$

No. 3 No. 4

No. 5

No. 6

BULBLETS

20.00

16.00

1.60

12.00

$\begin{array}{rr}. \ldots . & 1.25 \\ .10 & .08\end{array}$

.70

5.00

.60

40.00

4.00

32.00

.10
.70

5.00

.08
.60

4.00

32.00

.15

1.25

8.00

.12

1.00

7.00

.08

.60

4.00

.60

.50
3.00

4.50

.50

4.00

.07

.50

3.00

24.00

.07

3.00

25.00

.10

.80
6.00

.07

2.40

.40

3.00

.40

2.00

16.00

.40

2.50

22.00

.60

4.00

.50

.10

.60

.40

.35

.50

4.00

.10

3.00

.40

2.40

24.00

20.00

1.80

1.80
.30

2.40

.40

.50

5.00

4.00

3.00

40.00

32.00

24.00

.10

1.00

.80

.70

.30

.30

1.60

15.00

.30

2.20
18.00

.50

.30

.40

.50

.10

.50

40

3.00

2.40

.20
.30
.30
1.60

1.50

1.25

1.00

.50

.60

3.20

25.00

2.40
18.00

.40

1.60

12.00

.10

.60

.10

.......

.08

.70

.25

.30

2.50

2.00

.06

.40
.07

.60

3.00

.20
1.50

$\ldots .$.
$\ldots \ldots$
.50
2.50
.15
1.25
$\ldots \ldots$
.50

1.00

.80

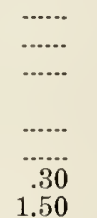

12.00

.25

1.50

12.00

.50

3.00

......

20

1.60

.25

.20

1.20

11.00

.20

1.40

12.00

.40

.15

.20

1.20

.10
.....

.75

.30

1.20
9.00

......

.....

2.00

.10
.90

.40

1.00

.15

1.00

8.00

.40

2.00

…...

10

1.20

.20

7.00

.10

1.00

.30

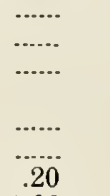

1000

Qt.

1.00

8.00

1000

Qt.

......

1000

1000

1.00

10

1000

10

2.40

20.00

.10

1000

.25

8.00

Qt.

1.00

…....

1000

Qt.

1000

1.00

10

1.50

3.00

Mixtures-While we do not grow any mixtures or unnamed seedlings we will make up Special Mixtures giving extra value to those who do not care for the names as follows:

Special Mixtures-Large (blooming size bulbs) per $10, \$ 0.40$; per $100, \$ 3.50$ 